\title{
Collocation Method for Axisymmetric Bending Problems of Circular Thin Plates Based on Barycentric Interpolation
}

\author{
Fuhe Ge, Meiling Zhuang and Changqing Miao* \\ Key Laboratory of Concrete and Prestressed Concrete Structures of Ministry of Education, Southeast University, Nanjing, China \\ School of Civil Engineering, Southeast University, Nanjing, China \\ ${ }^{*}$ Corresponding author
}

\begin{abstract}
Collocation method based on barycentric rational interpolation (BRICM) is used in this article to study axisymetric bending problems of circular thin plates in polar coordinates. Unknown function is approximated by barycentric rational interpolation and the discrete algebraic equations of differential equations are obtained by forcing the differential equations to satisfy the value of the discrete nodes. The boundary conditions are imposed by substitution method and the numerical solution can be obtained by solving the differential equations. Numerical calculation example demonstrates that the proposed method for circular thin plates bending problems has the merits of higher computation accuracy, convenient program, simple calculation formulations.
\end{abstract}

Keywords-axisymetric bending problems; polar coordinates; barycentric rational interpolation; collocation method

\section{INTRODUCTION}

Circular plates are common in many structures such as nozzle covers, end closures in pressure vessels, pump diaphragms, turbine disks, and bulkheads in submarines and airplanes, etc [1]. The analytic method used to solve circular plate differential equation is impossible in many engineering designs and the engineering experiment is expensive and complicated. It is needed to study a high accuracy numerical method for analysis of bending of circular plate. When circular plates are analyzed, it is convenient to express the governing differential equation in polar coordinates.

At present, there are many methods to obtain the numerical solution of bending of circular plate such as finite difference method [2], finite element method [3], boundary element method [4], meshless method [5], differential quadrature method [6], Fourier differential quadrature method [7], homotopy perturbation method (HPM) [8], etc.

The meshless method also named as element free method (EFM) only needs nodal data and not date of element [5]. The current popular meshless method is that the algebraic equation of problem can be obtained through the approximate smooth function produced by the moving least square method [9]. The computation of EFM is very large. And it is more difficult to satisfy the essential boundary condition and discontinuity condition of the material because of the feature that the the approximation function of EFM does not pass through variable values of the nodes. Differential quadrature method is mainly used in nonlinear analysis and multidimensional domain by using little computation to obtain high precision result. But it is difficult to solve the problem with irregular region and it has instability to a large number of nodes by using differential quadrature method [6]. The computation of Fourier differential quadrature method is very large [7]. Based on the selected differential equations which have analytical solution, numerical solution can be obtained by HPM [8].

Although the above methods can solve the bending of plate, they have some disadvantages such as lacking of flexibility and a low accuracy [10].

Based on barycentric interpolation collocation method [11], this paper has established a barycentric rational interpolation collocation method (BRICM) for solving the axisymmetric bending problems of circular thin plate, and uses numerical example to verify effectiveness and computational accuracy of this proposed method. Due to the symmetry of circular thin plate, the two-dimensional problem is transformed into onedimension problem, which greatly reduces the work time and improves the work efficiency.

Numerical calculation example show that BRICM has advantages of convenient use, higher efficiency, and higher accuracy compared with homotopy perturbation method (HPM) [10], meshless method and finite element method (FEM).

\section{BRICM AND ITS DIFFERENTIAL MATRICES}

First, given a function defined on the interval and the function values on the nodes are $u_{i}=u\left(r_{i}\right), i=1,2, \cdots, n$, the barycentric rational interpolation [12] of function is

$$
u(r)=\sum_{j=1}^{n} \frac{w_{j}}{r-r_{j}} u_{j} / \sum_{j=1}^{n} \frac{w_{j}}{r-r_{j}}
$$

Where $w_{j}=\sum_{i \in J_{j}}(-1)^{i} \prod_{k=i, k \neq j}^{i+d} \frac{1}{\left(r_{j}-r_{k}\right)}, j=1,2, \cdots, n$ is the barycentric rational interpolation weight, $J_{j}=\{i \in I: j-d \leq i \leq j\}, d=1,2, \cdots n$ is an index set.

The barycentric rational interpolation of function $u(r)$ can be simplified as 


$$
u(r)=\sum_{j=1}^{n} L_{j}(r) u_{j}, L_{j}(r)=\frac{w_{j}}{r-r_{j}} / \sum_{k=1}^{n} \frac{w_{k}}{r-r_{k}}
$$

Where $L_{j}(r)=\frac{w_{j}}{r-r_{j}} / \sum_{k=1}^{n} \frac{w_{k}}{r-r_{k}}, j=1,2, \cdots, n$ is the barycentric rational interpolation basis function.

Then the mth-order derivative of function can be written as

$$
u^{(m)}(r)=\frac{d^{m} u(r)}{d x^{m}}=\sum_{j=1}^{n} L_{j}^{(m)}(r) u_{j}
$$

The mth-order derivative of function $u(r)$ on the nodes $r_{1}, r_{2}, \cdots, r_{n}$ can be written as

$$
u^{(m)}\left(r_{i}\right)=\sum_{j=1}^{n} L_{j}^{(m)}\left(r_{i}\right) u_{j}=\sum_{j=1}^{n} D_{i j}^{(m)} u_{j}, \quad i=1,2, \cdots, n
$$

In (4), $D_{i j}^{(m)}=L_{j}^{(m)}\left(r_{i}\right)$ represents the value of the mth-order derivative of function $L_{j}(r)$ on the node $r_{i}$. Equation (4) can be written in the following matrix form[13]:

$$
\boldsymbol{u}^{(m)}=\boldsymbol{D}^{(m)} \boldsymbol{u}
$$

In (5), matrix $\boldsymbol{D}^{(m)}$ is $m$ th-order barycentric rational interpolation differential matrix on nodes $r_{1}, r_{2}, \cdots, r_{n}$, which is composed of the elements $D_{i j}^{(m)}=L_{j}^{(m)}\left(x_{i}\right)$. $u^{(m)}=\left[u_{1}^{(m)}, u_{2}^{(m)}, L, u_{n}^{(m)}\right]^{T}$ and $\boldsymbol{u}=\left[u_{1}, u_{2}, \cdots, u_{n}\right]^{T}$ represent the column vector of $m$ th-order derivative value and the value of the function $u(r)$ on the nodes, respectively.

\section{BRICM FOR AXISYMMETRIC BENDING OF CIRCULAR Thin Plate in Polar CoORdinates}

The governing equation of the circular plate axisymmetric bending of circular thin plates [14] in polar coordinates is as following:

$$
\nabla_{r}^{4} u \equiv\left(\frac{d^{2}}{d r^{2}}+\frac{1}{r} \frac{d}{d r}\right)\left(\frac{d^{2} u}{d r^{2}}+\frac{1}{r} \frac{d u}{d r}\right)=\frac{q}{D}
$$

And in the extended form can be written as

$$
\frac{d^{4} u}{d r^{4}}+\frac{2}{r} \frac{d^{3} u}{d r^{3}}-\frac{1}{r^{2}} \frac{d^{2} u}{d r^{2}}+\frac{1}{r^{3}} \frac{d u}{d r}=\frac{q}{D}
$$

Where $u=u(r)$ is the unknown deflection, $q$ is uniform load, $D=E t^{3} / 12\left(1-v^{2}\right)$, with $E$ Young's modulus, $t$ the thickness of thin plate and $v$ is Possion's ratio of elasticity.

The three boundary conditions of circular thin plate including clamped boundary, simply-supported boundary and free boundary are represented by $\Gamma_{1}, \Gamma_{2}$ and $\Gamma_{3}$ respectively in polar coordinates. $\Gamma=\partial \Omega=\Gamma_{1} \cup \Gamma_{2} \cup \Gamma_{3}$ is the boundary of the domain $\Omega$ of circular thin plate with the boundary condition

$$
B u \stackrel{\Delta}{\Delta} \begin{cases}u=0 & \text { on } \Gamma_{1} \cup \Gamma_{2} \\ C u=0 & \text { on } \Gamma_{1} \\ S u=0 & \text { on } \Gamma_{2} \cup \Gamma_{3} \\ F u=0 & \text { on } \Gamma_{3}\end{cases}
$$

In (8), C, S, F are defined as

$$
C=\frac{d}{d r}, S=\frac{1}{r} \frac{d}{d r}+v \frac{d^{2}}{d r^{2}}, F=\frac{d^{3}}{d r^{3}}+\frac{1}{r} \frac{d^{2}}{d r^{2}}-\frac{1}{r^{2}} \frac{d}{d r}
$$

(6) can be transformed to barycentric rational interpolation form by (5)

$$
\left(\boldsymbol{D}^{(4)}+\operatorname{diag}(2 / r) \boldsymbol{D}^{(3)}-\operatorname{diag}\left(1 / r^{2}\right) \boldsymbol{D}^{(2)}+\boldsymbol{D}^{(1)}\right) \boldsymbol{U}=\boldsymbol{q} / D(10)
$$

where $\boldsymbol{U}=\left[u_{1}, u_{2}, \ldots, u_{n}\right]^{T}, \boldsymbol{q}=\left[q_{1}, q_{2}, \ldots, q_{n}\right]^{T}$.

(8) can be transformed to barycentric rational interpolation collocation form by (4)

$$
\begin{aligned}
& \Gamma_{1}: \sum_{j=1}^{n} D_{i j}^{(0)} u_{j}=0, \sum_{j=1}^{n} D_{i j}^{(1)} u_{j}=0 \\
& \Gamma_{2}: \sum_{j=1}^{n} D_{i j}^{(0)} u_{j}=0, \frac{1}{r} \sum_{j=1}^{n} D_{i j}^{(1)} u_{j}+v \sum_{j=1}^{n} D_{i j}^{(2)} u_{j}=0 \\
& \Gamma_{3}: \frac{1}{r} \sum_{j=1}^{n} D_{i j}^{(1)} u_{j}+v \sum_{j=1}^{n} D_{i j}^{(2)} u_{j}=0, \sum_{j=1}^{n} D_{i j}^{(3)} u_{j}+\frac{1}{r} \sum_{j=1}^{n} D_{i j}^{(2)} u_{j}-\frac{1}{r^{2}} \sum_{j=1}^{n} D_{i j}^{(1)} u_{j}=0
\end{aligned}
$$

(11) can be written in the following matrix form:

$$
\begin{array}{ll}
\Gamma_{1}: & \boldsymbol{B}_{0} \boldsymbol{U}=0, \boldsymbol{B}_{1} \boldsymbol{U}=0 \\
\Gamma_{2}: & \boldsymbol{B}_{0} \boldsymbol{U}=0, \boldsymbol{B}_{2} \boldsymbol{U}=0 \\
\Gamma_{3}: & \boldsymbol{B}_{2} \boldsymbol{U}=0, \boldsymbol{B}_{3} \boldsymbol{U}=0
\end{array}
$$

Boundary conditions are imposed to the algebraic equations with substitution method [15]. The barycentric rational interpolation collocation matrix of circular thin plate in polar coordinates can be obtained with clamped boundary (a), simply-supported boundary (b) and free boundary (c).

$$
\text { (a): }\left[\begin{array}{l}
\boldsymbol{L} \\
\boldsymbol{B}_{0} \\
\boldsymbol{B}_{1}
\end{array}\right],\left(\begin{array}{l}
\boldsymbol{F} \\
\boldsymbol{0} \\
\boldsymbol{0}
\end{array}\right), \quad(b):\left[\begin{array}{l}
\boldsymbol{L} \\
\boldsymbol{B}_{0} \\
\boldsymbol{B}_{2}
\end{array}\right] \boldsymbol{U}=\left(\begin{array}{l}
\boldsymbol{F} \\
\boldsymbol{0} \\
\boldsymbol{0}
\end{array}\right), \quad(c):\left[\begin{array}{l}
\boldsymbol{L} \\
\boldsymbol{B}_{2} \\
\boldsymbol{B}_{3}
\end{array}\right] \boldsymbol{U}=\left(\begin{array}{l}
\boldsymbol{F} \\
\boldsymbol{0} \\
\boldsymbol{0}
\end{array}\right)
$$

Where $\boldsymbol{L}=\left(\boldsymbol{D}^{(4)}+\operatorname{diag}(2 / r) \boldsymbol{D}^{(3)}-\operatorname{diag}\left(1 / r^{2}\right) \boldsymbol{D}^{(2)}+\boldsymbol{D}^{(1)}\right)$. The numerical solutions of the bending of the circular plate in polar coordinates can be obtained by solving (13). 


\section{NUMERICAL SOLUTION}

One numerical calculation example is given in this article to verify the effectiveness and computational accuracy of BRICM. The calculation program is compiled by MATLAB. By use of BRICM and Chebyshev node, the numerical solution is obtained and compared with analytical solution. $\boldsymbol{u}^{c}, \boldsymbol{u}^{e}$ are numerical solution column vector and analytical solution column vector, respectively. $E_{r}=\left\|\boldsymbol{u}^{c}-\boldsymbol{u}^{e}\right\|_{2} /\left\|\boldsymbol{u}^{e}\right\|_{2}$, $E_{a}=\left\|\boldsymbol{u}^{c}-\boldsymbol{u}^{e}\right\|_{2}$ are the relative error and absolute error respectively between numerical solution and analytical solution.

As shown in Figure 1 , the radius is $a=5 \mathrm{~m}, t=1 \mathrm{~m}$, $E=2.0 \times 10^{11} \mathrm{~N} / \mathrm{m}^{2}, p=6.0 \times 10^{6} \mathrm{~N}$.

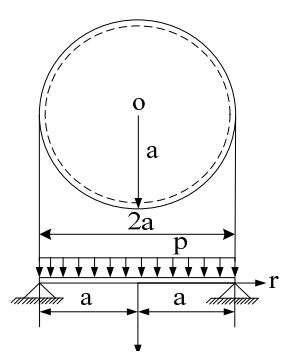

FIGURE I. DIAGRAM OF SIMPLY SUPPORTED CIRCULAR PLATE UNDER UNIFORM LOAD

The analytical solution of this calculation example with simply-supported boundary is

$$
u^{e}(r)=\frac{p\left(a^{2}-r^{2}\right)}{64 D}\left(\frac{5+v}{1+v} a^{2}-r^{2}\right) .
$$

For the calculation, computational domain is $\Omega=[0,5]$ and 11 nodes are selected in the direction of $r$. The boundary is $\Gamma_{2}$ in (11) and $u_{j}=u\left(r_{j}=5\right), j=1,2, \ldots, 11$. By solving the (a) in (13), the BRICM numerical solution of bending of the plate can be obtained.

We can know that the numerical solution of FEM has a low accuracy and the numerical solution of HPM is highly consistent with analytical solution from the analysis of Fig.3(FEM result and HPM result compared with EXACT result) in [8]. Figure 2 shows that the results of BRICM and HPM are both highly consistent with EXACT results. Figure 3 shows that the accuracy of the BRICM result compared with EXACT result is $10^{-12}$.

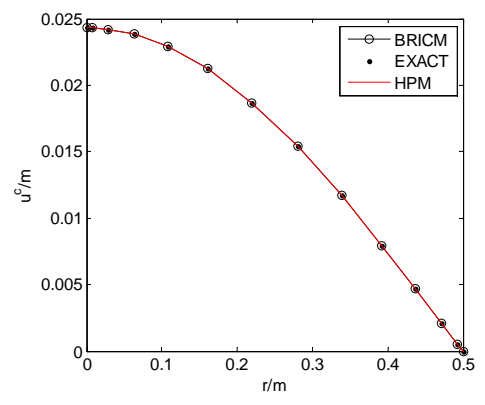

FIGURE II. BRICM AND HPM NUMERICAL RESULTS AND EXACT RESULT

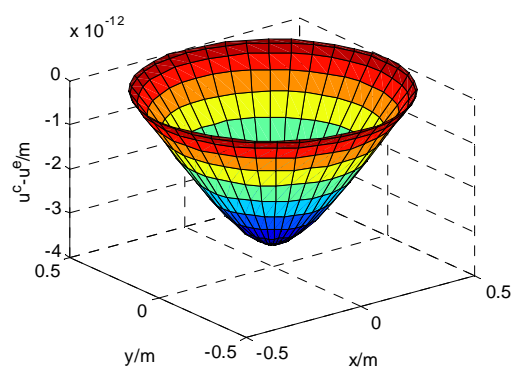

FIGURE III. THE ABSOLUTE ERROR BETWEEN BRICM RESULTS AND EXACT RESULTS

Based on the numerical results of Table 1 and Table 2 comprehensively, it can be summed up as follows: BRICM has higher computation accuracy with $10^{-11}$ than FEM with $10^{-2}$ and meshless method with $10^{-3}$.

TABLE I. THE RELATIVE ERROR OF MESHLESS METHOD RESULT OF SIMPLY-SUPPORTED CIRCULAR PLATE IN [16]

\begin{tabular}{|c|c|c|c|c|}
\hline $\begin{array}{c}\text { Number of } \\
\text { nodes }\end{array}$ & $r / a=0$ & $r / a=0.1$ & $r / a=0.2$ & $r / a=0.5$ \\
\hline 10 & $1.4000 \times 10^{2}$ & $1.2600 \times 10^{-2}$ & $1.2100 \times 10^{-2}$ & $1.2000 \times 10^{-2}$ \\
\hline 20 & $3.3000 \times 10^{-3}$ & $3.1800 \times 10^{-3}$ & $2.9700 \times 10^{-3}$ & $3.1200 \times 10^{-3}$ \\
\hline 40 & $2.6700 \times 10^{-3}$ & $2.5400 \times 10^{-3}$ & $2.3100 \times 10^{-3}$ & $1.7800 \times 10^{-3}$ \\
\hline
\end{tabular}

TABLE II. THE RELATIVE ERROR OF DIFFERENT COMPUTATION METHODS WITH DIFFERENT NODES OF THE EXAMPLE $(\mathrm{R} / \mathrm{A}=0.5)$

\begin{tabular}{|c|c|c|c|}
\hline Number of nodes & BRICM & Meshless Method & FEM \\
\hline 10 & $5.3439 \times 10^{-11}$ & $1.2000 \times 10^{-2}$ & \multirow{2}{*}{$1.0370 \times 10^{-2}$} \\
\hline 20 & $8.9192 \times 10^{-9}$ & $3.1200 \times 10^{-3}$ & \multirow{2}{*}{} \\
\hline 40 & $2.0979 \times 10^{-7}$ & $1.7800 \times 10^{-3}$ & \\
\hline
\end{tabular}

\section{CONCLUSIONS}

In this article, BRICM is proposed for solving the bending of circular thin plate in polar coordinates. The method has simple calculation formulations and the calculation program is compiled by MATLAB. It is effective and convenient to many engineering designers to solving similar problem. Numerical example demonstrate that the proposed method has high computation accuracy with $10^{-11}$ and with the increasing of the number of nodes the accuracy is between $10^{-7}$ and $10^{-9}$. Compared with FEM, BRICM has higher computation accuracy, simple calculation formulations and dispenses with meshing and transforming irregular domain into a regular 
domain according the coordinate transformation. The method has provided a new meshless method with high precision for bending problem of plate in engineering and is worth to be generalized to irregular plate problem and other engineering problems which need high accuracy.

\section{ACKNOWLEDGMENT}

This research has been supported by the National Natural Science Foundation of China under grant number 51778135 and Aeronautical Science Foundation of China under grant number 20130969010.This research also has been supported by the Fundamental Research Funds for the Central Universities and Postgraduate Research \& Practice Innovation Program of Jiangsu Province under grant No. KYCX18_0113.

\section{REFERENCES}

[1] Kishor R. G., Kirtiwant P. G.. An Inverse Problem for the Quasi-static Thermoelastic System in a Thin Clamped Circular Plate, J. Advances in Applied Mathematical Analysis, 2011, 6:43-54.

[2] Ravari M R K, Talebi S, Shahidi A R. Analysis of the buckling of rectangular nanoplates by use of finite-difference method, J. Meccanica, 2014, 49(6):1443-1455.

[3] Kwon Y W, Bang H. The finite element method using MATLAB (2nd ed.). CRC Press, 2015.

[4] Tanaka M., Matsumoto T., Oida S.. A boundary element method applied to the elastostatic bending problem of beam-stiffened plates[J]. Engineering Analysis with Boundary Elements, 2000, 24(10): 751-758.

[5] Pardo E. Meshless method for linear elastostatics based on a path integral formulation[J]. International Journal for Numerical Methods in Engineering, 2015, 47(8):1463-1480.

[6] Striz A G, Chen W, Bert C W. Static analysis of structures by the quadrature element method (QEM)[J]. International Journal of Solids \& Structures, 2013, 31(20):2807-2818.

[7] Wenting Shao, Xionghua Wu. Fourier differential quadrature method for irregular thin plate bending problems on Winkler foundation[J]. Engineering Analysis with Boundary Elements ,2011, 35:389-394.

[8] Rostamiyan Y., Fereidoon A., Davoudabadi M. R. et al. Anzlytical approach to investigation of deflection of circular plate under uniforn load by homotopy perturbation method [J]. Mathematical and Computational Applications, 2010, 15( 5):816-821.

[9] Hale, Brand J S. Meshless methods for shear-deformable beams and plates based on mixed weak forms[J]. Imperial College London, 2013.

[10] Zhaoqing W., Shuchen L., Jian J., Yang P.. A Highly Accurate Regular Domain Collocation method for Solving Potential Problems in the Irregular Doubly Connected Domains, Mathematical Problems in Engineering, 2014

[11] Syed T M,Ahmet Y,Hosseini M M. An iterative algorithm for fifth-order boundary value problems. Word Applied Sciences Joural ,2010,10(2):222-230.

[12] Berrut, J. P., Klein, G. Recent advances in linear barycentric rational interpolation .Journal of Computational \& Applied Mathematics,2014, 259(4), 95-107.

[13] Güttel, S., Klein, G. Convergence of linear barycentric rational interpolation for analytic functions. Siam Journal on Numerical Analysis,2012,50(5), 2560-2580.

[14] Ventsel E, Krauthammer T, Carrera E. Thin Plates and Shells: Theory, Analysis, and Applications[J]. Applied Mechanics Reviews, 2002, 55(4):1813-1831.

[15] Zhang L W, Li D M, Liew K M. An element-free computational framework for elastodynamic problems based on the IMLS-Ritz method[J]. Engineering Analysis with Boundary Elements, 2015, 54:3946.

[16] Ng T Y, Li H, Cheng J Q, et al. A novel true meshless numerical technique (HM-DOR method) for the deformation control of circular plates integrated with piezoelectric sensors/actuators[J]. Smart Materials \& Structures, 2003, 12(6):955. 